\title{
Gastric perforation by fish bone: Case report
}

\begin{abstract}
Summary
Introduction: The ingestion of foreign bodies is usually accidental and asymptomatic, it is observed more frequently in patients with psychiatric diseases, children and older adults, being able to cause gastric perforation in $1 \%$ of cases. Clinical case: A 70 -year-old male patient who comes to the emergency due to severe pain located in the epigastrium, with the presence of a palpable, painful mass with irregular edges. Discussion: the preoperative diagnosis of hollow viscera perforation by a foreign body must take into account the epidemiological history, directed interrogation, physical examination and paraclinical studies to allow its differentiation with other medical or surgical entities, allowing the appropriate preoperative and intraoperative management of the patient. Conclusion: In spite of the infrequent, the perforation of hollow viscera by foreign body should be a differential diagnosis to be considered by the general surgeon.
\end{abstract}

Keywords: epigastrium, intraoperative management, psychiatric diseases, asymptomatic
Volume 10 Issue 5 - 2019

\author{
Pedro Monsalve, ' Gabriela Lombardo \\ Pascarelli,' Elaine J Bastardo Milano \\ 'Specialist Surgeon, Central University of Venezuela, Venezuela \\ ${ }^{2}$ Instructor Professor, Central University of Venezuela,Venezuela
}

Correspondence: Elaine J Bastardo Milano, Instructor professor. Universidad Central de Venezuela. Active Member SVC,Venezuela,Email elainebm05@gmail.com

Received: September 21, 2019 | Published: October 25, 2019

\section{Introduction}

Most of the foreign bodies ingested are digested and expelled without causing symptoms, of the remaining percentage (depending on the shape and size) less than $1 \%$ remain in the stomach and can cause perforation and affect adjacent organs such as the pancreas. ${ }^{1}$ This is usually manifested as an intra-abdominal abscess in $71 \%$ of cases, with symptoms that may occur within days and even weeks after ingestion and whose etiology is a mystery to the surgical team. Although these patients are studied exhaustively with radiological and endoscopic techniques, it is not until the same surgical act where the final diagnosis is made, already when generally very aggressive approaches have been made. ${ }^{2,3}$ In the present article we report the case of a patient who debuted with a palpable abdominal tumor in the epigastrium who was initially diagnosed and managed as a pancreatic head tumor, and who after performing a diagnostic laparoscopy showed gastric perforation by fishbone.

\section{Case presentation}

A 70-year-old male patient who consulted for burning abdominal pain of progressive intensity in epigastrium and multiple febrile hooks of $39^{\circ} \mathrm{C}$. On physical examination: loss of $2.5 \mathrm{~kg}$ compared to your usual weight and flat abdomen, not irritated, but painful to deep palpation in epigastrium, with the presence of poorly defined edge mass and $6 \mathrm{~cm}$ diameter. In the laboratory profile, leukocytosis with a deviation to the left was evidenced, with elevation of alkaline phosphatase (ALP), amylase and lipase within normal limits, with serologies and negative tumor markers (CA-19-9, CEA, AFP).

Abdominal ultrasound Figure 1 showed a cystic-looking image with low-level, ecolucent images inside, $20 \times 13 \mathrm{~mm}$ in diameter, while in double-contrast computed tomography (Figure 2) observed a lesion occupying retrogastric space, towards the transcavity of the epiplons, adjacent to the pancreatic head, hypodense, with poorly defined edges, with attenuation coefficients within the solid and liquid range, $5.6 \times 5.6 \mathrm{~cm}$ in diameter. In the upper digestive endoscopy a rounded lesion was evident that pushed the posterior face of the heart.
A puncture was performed and a purulent liquid was drained from which sample was taken for cytology and culture, which proved to be an inflammatory exudate with abundant polymorphonuclear and basophilic bacterial aggregates (Actynomices). The patient is managed with medical treatment and antibiotic therapy for several weeks under the presumptive diagnosis of complicated pancreatic head tumor with intra-abdominal collection; however, in the persistence of the symptomatology, a diagnostic laparoscopy was planned. During the surgical act when addressing the transcavity of the epiplons, a foreign body was found that came from the stomach (Figure 3), perforating its posterior wall and making contact with the head of the pancreas conditioning a plastron (Figure 4). The foreign body was removed and after a wash and aspiration of the cavity, the surgery was completed.

When the foreign body was examined, it was determined that it was a fish bone, information that was corroborated by the patient when it was reinterrogated and evidenced retrospectively in the tomography performed. The patient presented immediate clinical improvement and after satisfactory evolution he was discharged without eventualities.

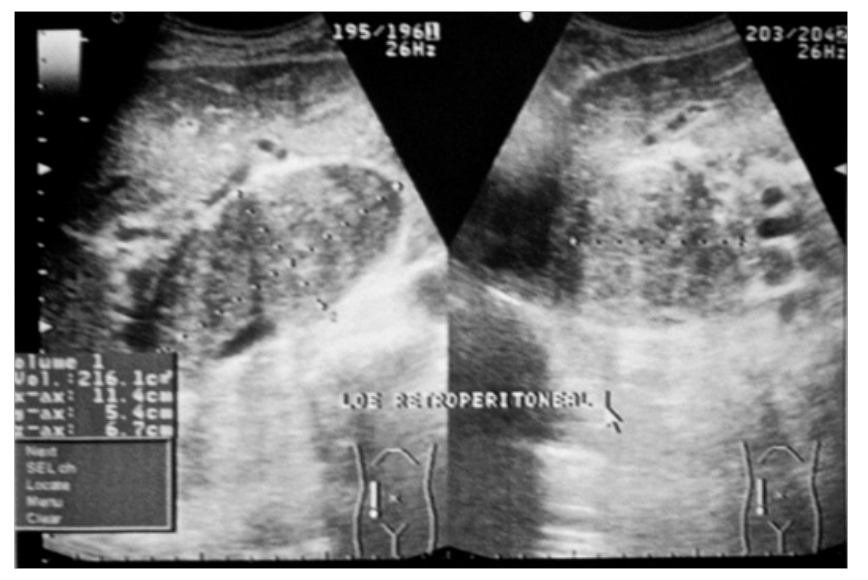

Figure I Abdominal ultrasound. The yellow arrows indicate the location of the retroperitoneal collection image. 


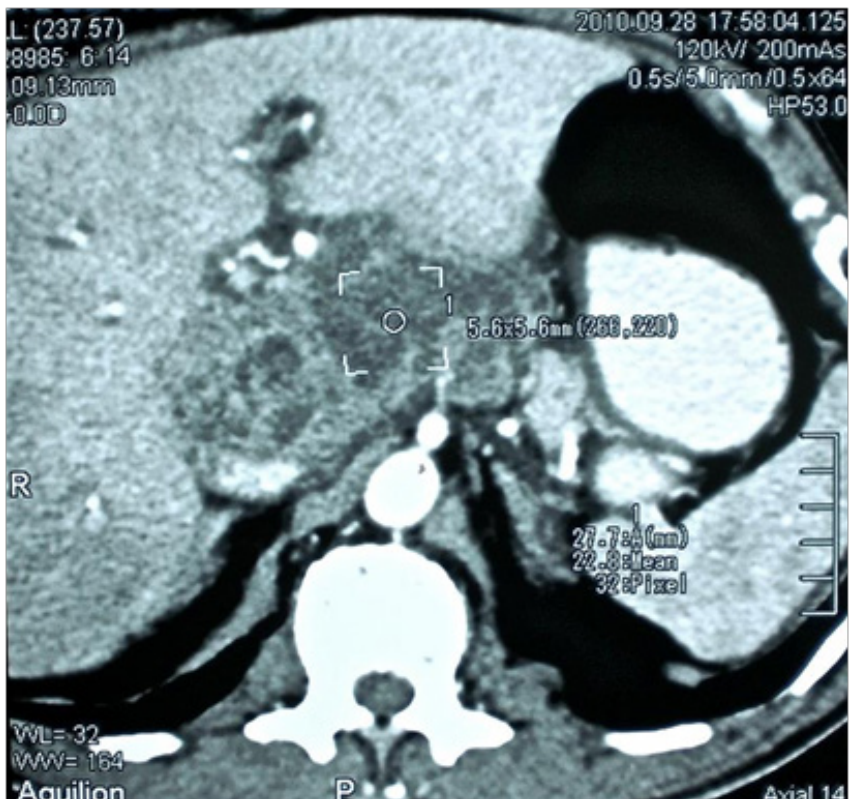

Figure 2 Computerized axial tomography with double contrast. Yellow arrow points retroperitoneal collection image of heterogeneous appearance.

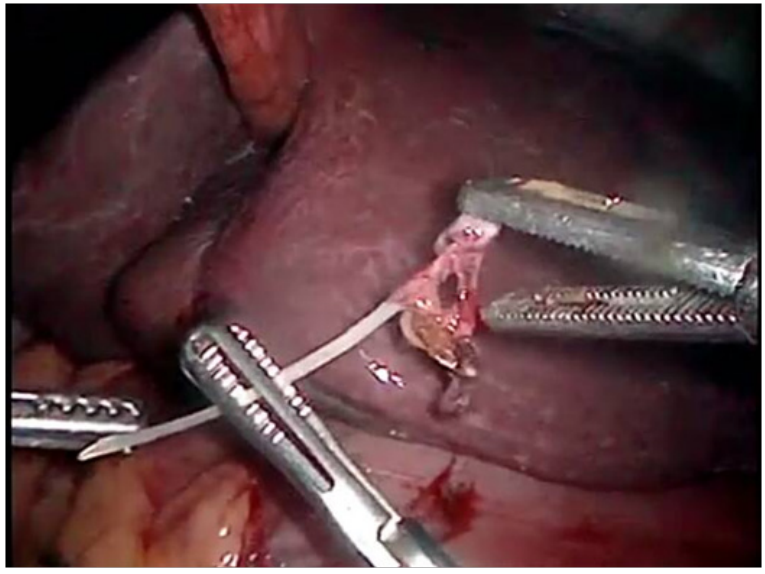

Figure 3 Laparoscopic image showing fish bone in the transcavity of the epiploons (indicated by yellow arrow).

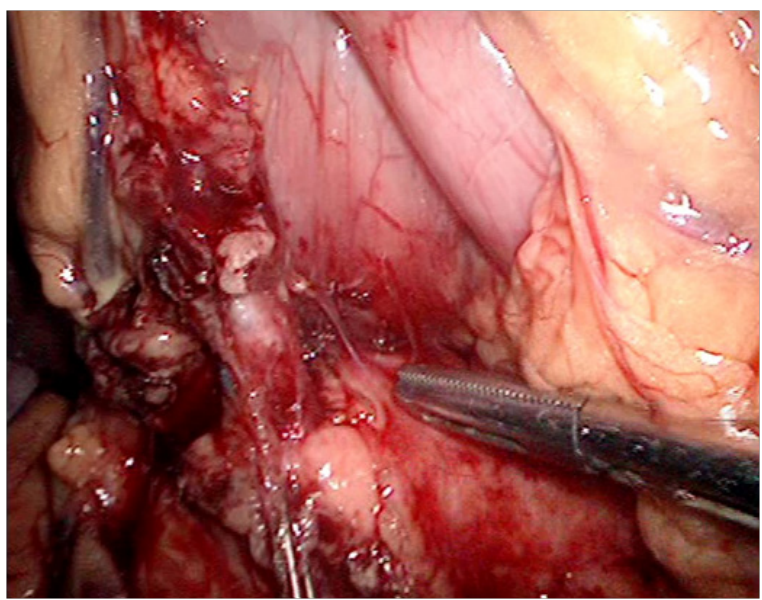

Figure 4 Laparoscopic image showing plastron between gastric posterior wall and pancreas head.

\section{Discussion}

Perforations of the gastrointestinal tract caused by foreign bodies are not commonly diagnosed in the preoperative evaluation due to the non-specificity of the symptoms. Clinically it presents as peritonitis, intra-abdominal abscesses, obstruction, and fistulas among others ${ }^{4}$. Up to $23 \%$ of cases, the correct preoperative diagnosis is made, confusing in the rest of the cases with pancreatic carcinoma, acute cholecystitis or gastric stromal tumors (GIST). ${ }^{5,6}$

For the differential diagnosis it is required to thoroughly investigate the medical and epidemiological history of the patient, anamnesis and physical examination, imaging findings, laboratory tests and analysis of selective tumor markers such as AFP, CA 19-9 and CEA; The most commonly used tumor marker to identify a pancreatic neoplasm is CA 19-97. Liver enzymes are usually within normal or occasionally high limits. The people with the highest risk of ingestion of foreign bodies are patients with psychiatric diseases such as schizophrenia and depression, also alcoholics, children and older adults. ${ }^{4,5}$ Most perforations occur in the narrow areas and angles of the digestive tract, such as the pyloric region, duodenum or ileocecal valve although they have also been reported in other organs such as bladder and liver. ${ }^{1,8}$ In the case presented, the patient showed only leukocytosis with an increase in ALP.

Gastric perforations are usually observed in the lesser curvature, the foreign body can partially perforate the wall, causing inflammatory changes and subsequently a perigastric abscess; In some cases a liver abscess may form when the entire anterior gastric wall or the first portions of the duodenum that are in contact with the left hepatic lobe penetrates. ${ }^{9}$ Recent studies have shown that liver abscess mortality is approximately $11-33 \%$ and the causative agent responsible for the most frequent inflammatory process is Streptococcus, however, in most cases it is unknown. ${ }^{10}$ When performing the upper digestive endoscopy, the lesion was observed on the back of the heart, in contact with the gastric wall, body of the pancreas and left hepatic lobe; In the cytomorphological findings according to the observed characteristics, compatibility with Actinomyces was obtained.

\section{Conclusion}

In spite of the infrequent, the perforation of hollow viscera by foreign body should be a differential diagnosis to be considered by the general surgeon. Although the history usually provides valuable data, many times they are not referred voluntarily by the patient, so the interrogation addressed to this diagnostic suspicion must be mandatory and thorough, thus allowing adequate preoperative and intraoperative management that guarantees the patient's well-being and avoiding unnecessary studies or procedures.

\section{Acknowledgements}

None.

\section{Conflicts of interest}

The authors declare there are no conflicts of interest related to the article.

\section{References}

1. Nunez Martinez O, Sanz Garcia C, Perez Enciso I, et al. Endoscopic resolution of gastric perforation with pancreas penetration by a fish bone. Gastroenterol Hepatol. 2017;41(9):567-568. 
2. Huang YH, Siao FY, Yen HH. Pre-operative diagnosis of pancreatic abscess from a penetrating fish bone. QJM. 2013;106(10):955-956.

3. Koch H. Operative endoscopy. Gastrointest Endosc. 1977;24(2):65-68.

4. Velitchkov NG, Grigorov GI, Losanoff JE, et al. Ingested foreign bodies of the gastrointestinal tract: retrospective analysis of 542 cases. World $J$ Surg. 1996;20(8):1001-1005.

5. Daniell SJ. Foreign body perforation of a jejunal diverticulum. $J$ R Soc Med. 1982;75(9):747-749.

6. Maleki M, Evans W. Foreign-body perforation of the intestinal tract. Report of 12 cases and review of the literature. Arch Surg. $1970 ; 101(4): 474-477$.
7. Kim SW, Song SK. Gastric pseudotumoral lesion caused by a fish bone mimicking a gastric submucosal tumor. J Gastric Cancer. 2014;14:204 206.

8. Beecher SM, O’Leary DP, McLaughlin R. Diagnostic dilemmas due to fish bone ingestion: Case report \& literature review. Int J Surg Case Rep. 2015;13:112-115.

9. Mesina C, Vasile I, Valcea DI, et al. Problems of diagnosis and treatment caused by ingested foreign bodies. Chirurgia. 2013;108(3):400-406.

10. Ali FE, Al-Busairi WA, Esbaita EY, et al. Chronic perforation of the sigmoid colon by foreign body. Curr Surg. 2005;62(4):419-422. 